\title{
Thermal Conductivity of Solid Materials
}

\section{Mohamed M Teamah ${ }^{\# 1}$, Mohamed M. Khairat Dawood ${ }^{\# 2}$, Ali I. Shehata ${ }^{\# 3}$, Mohamed M. Yassin ${ }^{\# 4}$}

\#1 Department of Mechanical Engineering, College of Engineering and Technology, Arab Academy for Science, Technology, and Maritime Transport, Abu-Quir, Alexandria, Egypt \#2 Department of Mechanical Engineering, Faculty of Engineering, Suez Canal University, Ismailia, Egypt

\#3 Department of Mechanical Engineering, College of Engineering and Technology, Arab Academy for Science, Technology, and Maritime Transport, Abu-Quir, Alexandria, Egypt \#4 Department of Mechanical Engineering, College of Engineering and Technology, Arab Academy for Science, Technology, and Maritime Transport, Abu-Quir, Alexandria, Egypt

Corresponding Author: Mohamed M.Yassin

\section{INTRODUCTION :}

Thermo physical properties such as specific heat, thermal conductivity and density are unique for any material. Within a conductive material, thermal conductivity is the main property in the thermal energy transfer. Specific heat and density are the important components involved in the analysis of energy and mass balances. When combining these three properties, we get the thermal diffusivity, which is used in the analysis of unsteady-state heat transfer.

Two major methods are used to measure the thermal conductivity of any material. The steady state method named guarded plate method which depends on a constant temperature difference achieved in the specimen of the material. This method needs complicated measurement system, it is unsuitable for the field application [1].

The transient (unsteady-state) techniques generally use a heater of a particular geometry inserted in the sample, and heated within a period of time. These measurement systems are less complex with respect to steady state methods and are better suited for field measurements. The experimental studies for determination of the thermal properties in a transient state has been conducted with numerous methods and techniques.

The linear heat source method relies on a steady heat source ,for example, a hot wire, that generate $\mathrm{s}$ a temperature field inside an infinite volume of a material. Based on the thermal conductivity of the desired material, the temperature rise in its sample will vary from one material to other. The thermal conductivity, then, can be calculated from the temperature rise at two unique times and the power of the heater. This method was used to measure the thermal conductivity of many solids, fluids and soil [2-4]. 
The introduction of high precision electronics such as Wheatstone bridge, led the researchers to calculate the temperature rise in the specimen from the difference of electrical resistance in the hot wire. J.W. Haarman [5] used this electronics evolution to eliminate the experimental errors in gases.

The hot wire method showed high accuracy in the measurement of the thermal conductivity of gases and liquids. To be eligible to measure any sort of material, J.J. Healy et al.[6] presented several corrections to the theory of the hot wire method such as wire properties, radiations, variable fluid properties.

In the recent years, the problem of the inescapable contact resistance between the specimen and the wire had been studied by M.V. Peralta-Martinez et al. used soft alumina layers [7-8] in molten metal at high temperatures, and by M.J. Assael et al. using a layer of soft silicone [9] with Pyroceram 9606 . The results showed precise results; however the inflexibility of the experimental setup, limits its applications.

One of the problems facing the previous technique is the positioning of the temperature element within fruits, S. Kumar et al.[10] studied several positions to obtain good results, but it led to a significant deviation from the standard values.

The hot wire technique suffers from the sophisticated designs and the difficulty of measuring some material such as building insulation materials and moist soils. A thermal conductivity probe (TCP) has been introduced by F.C. Hoope and F.R. Leeper [11]. The TCP differs from the hot wire as it combines the temperature sensor and the electrical wire in a protective aluminum enclosure.

A comparative study with the steady state method (guarded hot plate) has been conducted by D. D'eustachio and R. E. Schreiner to investigate the thermal conductivity of Silica gel and cellular glass [12]. The results insured a very good agreement.

Another conclusion has been reached by Pianella [13] et al.when comparing the thermal conductivity measurements of three green roof substrates, using the transient and the steady state methods. Two types of needle probes were used for the transient measurements, single and dual-needle. While for the steady state method a heat flow meter apparatus was used. The results showed that the values of the steady state method are more consistent and higher than the transient ones

A special TCP has been constructed to measure the thermal conductivity of small samples of white and dark meat by V.E. Sweat and C.G. Haugh[14].

The design of the TCP depends on the measured material condition. It may be as a hypodermic needle $(1.2 \mathrm{~mm})$ used by Chuan-Liang Hsu and Dennis R. Heldman to measure the thermal conductivity of aqueous starch [15] or much bigger $(10 \mathrm{~mm})$ if it will be used in situ as used by L. Perez et al. [16].

Recently numerical models have been presented by numerous researchers comparing the numerical and experimental values to determine the thermal conductivity of various materials.

J.K. Carson and J.P. Sekhon studied granular materials (sand and soil) at the solid phase [17] using Maxwell's conductivity model and solved by Microsoft excel solver function. W. Guo studied the bulk wood pellets thermal conductivity[18] solving a modified equation of the line heat source method by a MATLAB program.

The preparation of the sample specimen is always a major problem facing the measurements. A probe has been constructed by S. Hadiet al. [19] gel layer to eliminate the thermal contact resistance between the sample and the heat sensor. But, the results showed significant deviation, as it reached $35 \%$ especially for the thermal diffusivity values.

To predict the thermal conductivity of nanofluids, Zerradi et al. [20], introduced a new correlation of Nusselt number. The exiting values were taken from Lee et al. [21], where a transient hot wire technique - platinum wire of $76.2 \mu \mathrm{m}$ diameter and $180 \mathrm{~mm}$ of length- was 
used. A good agreement between the numerical and the experimental measurements was achieved.

To minimize the measurement time on planetary surfaces, an algorithm which reduces the data had been introduced by S. Nagiharaet al. [21]. When compared to the experimental values, the results showed a good agreement. However, this algorithm had the disadvantage of the necessity of the former knowledge of the density and the specific heat of the sample. Thermal contact resistance of an epoxy layer between the sample of the material and the TCP has been investigated by L. Dang and W. H. Leong through two mathematical models [22]. Which when compared to the experimental results of Ottawa sand obtained by V.R. Tarnawski et al. [23 - 25] revealed a negligible effect.

As the review shows the technique of obtaining the thermal properties, has its difficulties and limitations.

The aim of this research work is to represent a simple experimental technique to find the thermal conductivity of sand, calcium carbonate and cement.

\section{EXPERIMENTAL SET-UP:}

The aim of the experimental work presented is to obtain a temperature versus time history profile.

The experimental set-up consists of a container, heater and two thermocouple holders, a data logger and a DC power supply.

The material was held in a cylindrical container with $30 \mathrm{~cm}$ diameter and $20 \mathrm{~cm}$ height

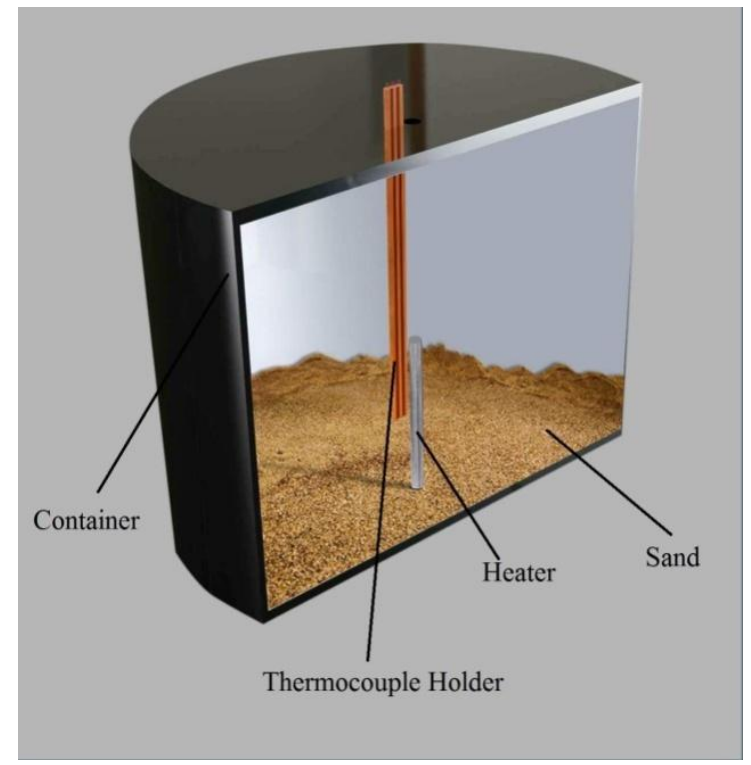

Fig.1Heater and TC holder in container

\section{Experimental Procedure:}

- The container was filled with the desired material.

- Start the experiment with applying DC current to the heater.

- Monitor all temperatures with the data logger. 


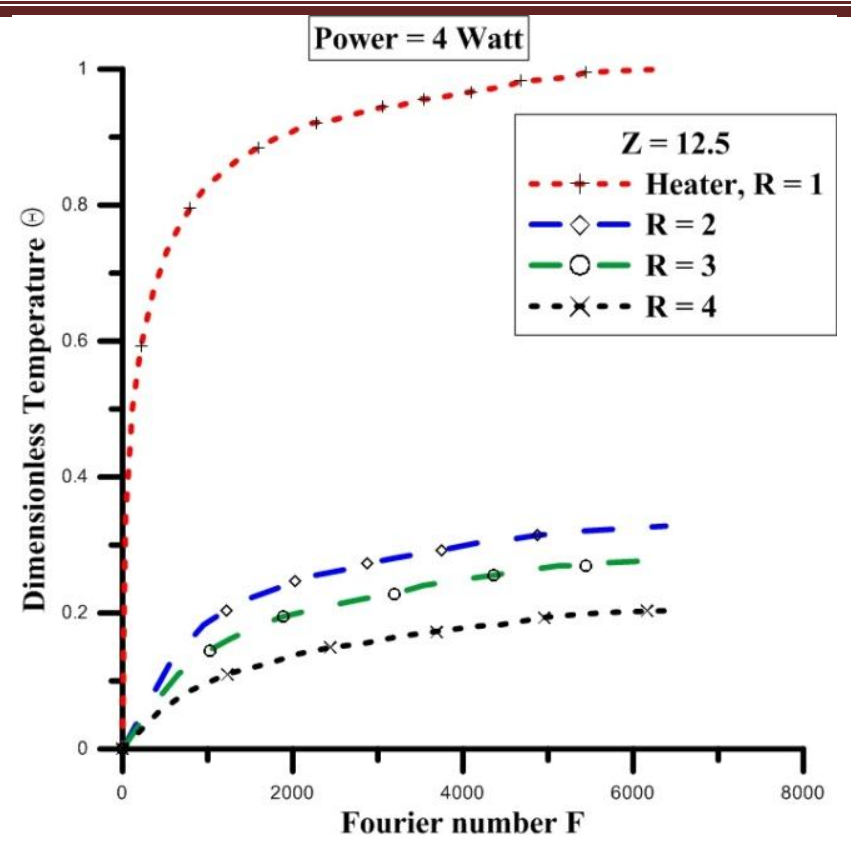

Fig.3 Dimensionless temperature vs. dimensionless time for sand, $\mathrm{P}=4 \mathrm{~W}$

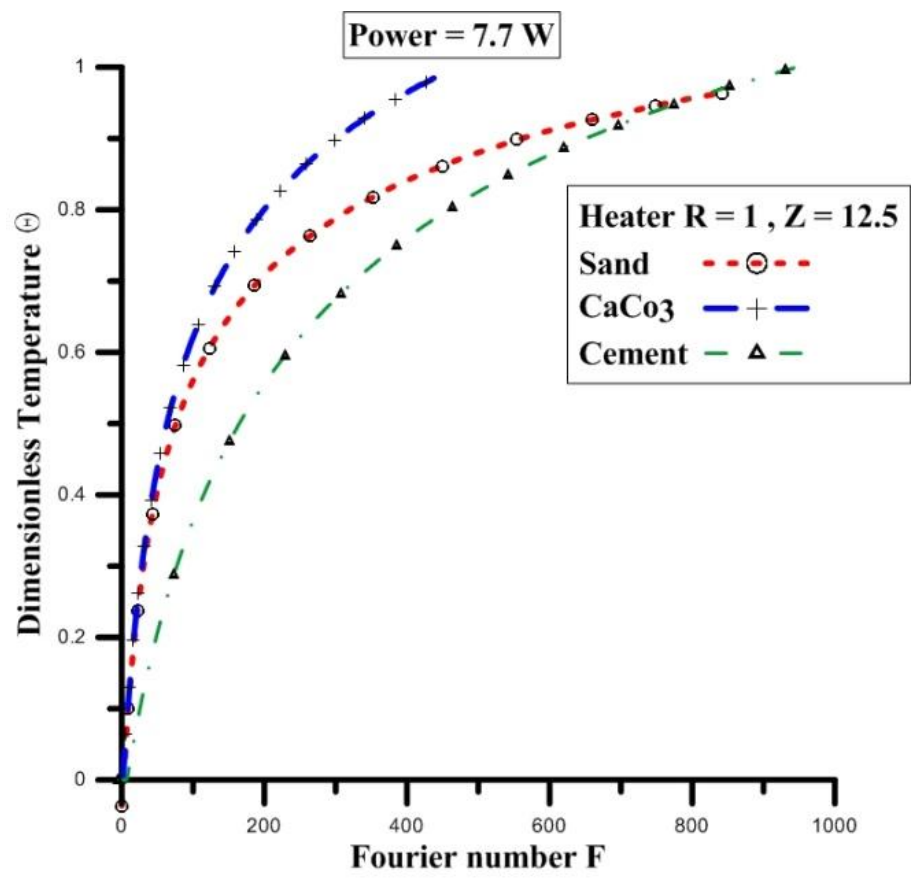

Fig. 4 Dimensionless temperature vs. dimensionless time for the heater in sand, calcium carbonate and cement, $\mathrm{P}=7.7 \mathrm{~W}$

\section{CONCLUSION}

The temperatures of the material are measured with respect to the temperature of the heater to obtain the dimensionless temperature.

The dimensionless radius and height are measured with respect to the heater radius.

The sand had a thermal diffusivity of $1.04 \mathrm{~mm}^{2} / \mathrm{s}$; The dimensionless time (Fourier Number) with the dimensionless temperature $(\Theta)$ in the horizontal direction was shown in Figure 3. 
The power produced by the heater was $4 \mathrm{~W}$. for the heater, the results showed that the temperature rises sharply with time then tends to a steady state. For the three thermocouples inserted in the sand, the same trend repeats itself.

The curve of the heater is far distant from the curves of the sand because the thermal diffusivity of the heater is four times bigger. The different positions of the thermocouples results in a distant curves.

Figure 4 illustrates the propagation of temperature when Calcium carbonate was used. The results showed higher response from the sand due to the higher thermal diffusivity.

\section{REFERENCES}

1. ASTM C177: Standard Test Method for Steady-State Heat Blux Measurements and Thermal Transmission Properties Ûy Means of the Guarded Hot-Plate Apparatus

2. E.F.M. van der Held, F.G. van Drunen, A Method of Measuring The Thermal Conductivity of Liquids, Physica 15, 865 (1949).

3. D.A. de Vries, A Nonstationary Method for Determining Thermal, Conductivity of Soil In Situ, Soil Sci. 73, 83 (1952).

4. A.G. Turnbull, Thermal Conductivity of Organic Silicates, J. Chem. Eng. Data 7, 79 (1962).

5. J.W. Haarman, Physica, A Contribution to the Theory of the Transient Hot-Wire Method, 52,605 (1971).

6. J.J. Healy, J.J. de Groot, J. Kestin, The Theory of The Transient Hot-Wire Method for Measuring Thermal Conductivity, Physica 82 C, 392 (1976).

7. M.V. Peralta-Martinez, M.J. Assael, M. Dix, L. Karagiannidis,W.A. Wakeham, A Novel Instrument for The Measurement of The Thermal Conductivity of Molten Metals, Part I: Instrument's Description, International Journal of Thermophysics, , 27, 353 (2006).

8. M.V. Peralta-Martinez, M.J. Assael, M. Dix, L. Karagiannidis,W.A. Wakeham, A Novel Instrument for the Measurement of the Thermal Conductivity of Molten Metals. Part II: Measurements, International Journal of Thermophysics, 27, 681 (2006).

9. M.J. Assael, K.D. Antoniadis, K.E. Kakosimos, I.N. Metaxa, An Improved Application of the Transient Hot-Wire Technique for the Absolute Accurate Measurement of the Thermal Conductivity of Pyroceram 9606 up to 420K, Int. J. Thermophys. 29, 445 (2008).

10. Shiva Kumar Modi, Durga Prasad B., Basavaraj M., Effect of moisture content and temperature on thermal conductivity of Psidiumguajava L. by line heat source method (transient analysis), International Journal of Heat and Mass Transfer 78 (2014) 354-359.

11. F.C. Hoope and F.R. Leeper, (1950), Transient Heat Flow Apparatus for the Determination of Thermal Conductivities, heating, piping and air conditioning.

12. D. D'eustachio and R. E. Schreiner, A Study Of A Transient Heat Method For Measuring Thermal Conductivity, of The American Society of Heating and Ventilating engineers, Spring Lake, N. J., June (1952).

13. Andrea Pianellaa, Robin E. Clarke, Nicholas S.G. Williams, Zhengdong Chen,Lu Aye, Steady-state and transient thermal measurements of green roofsubstrates, Energy and Buildings 131 (2016) 123-131.

14. V.E. Sweat and C.G. Haugh, A Thermal Conductivity Probe for Small Food Samples, Transaction of ASAE,56 (1974).

15. Chuan-Liang Hsu and Dennis R. Heldman, Prediction models for the thermal conductivity of aqueous starch, International Journal of Food Science and Technology (2004), 39, 737-743. 
16. L. Perez, B. Ladevie, P. Tochon, J.C. Batsale, A new transient thermal fouling probe for cross flow tubular heat exchangers, International Journal of Heat and Mass Transfer 52 (2009) 407-414.

17. J.K. Carson, J.P. Sekhon, Simple determination of the thermal conductivity of the solid phase of particulate materials, International Communications in Heat and Mass Transfer 37 (2010) 1226-1229.

18. Wendi Guo, C. Jim Lim, Xiaotao Bi, Shahab Sokhansanj, StaffanMelin, Determination of effective thermal conductivity and specific heat capacity of wood pellets, Fuel 103 (2013) 347-355.

19. SyamsulHadi, Mamoru Nishitani, Agung Tri Wijayanta, Takanobu Fukunaga , KosakuKurata , Hiroshi Takamatsu, Contact measurement of thermal conductivity and thermal diffusivity of solid materials: Experimental validation of feasibility with a prototype sensor, International Journal of Heat and Mass Transfer 69 (2014) 256-263.

20. Hicham Zerradi, Said Ouaskit, Aouatif Dezairi, Hamid Loulijat, Sofia Mizani, New Nusselt number correlations to predict the thermal conductivityof nanofluids, Advanced Powder Technology 25 (2014) 1124-1131.

21. S. Lee, S. U.-S. Choi, S. Li,J. A. Eastman, Measuring Thermal Conductivity of Fluids Containing Oxide Nanoparticles,Journal of Heat Transfer, Vol. 121, (1999) 280-289.

22. Seiichi Nagihara, Magnus Hedlund, Kris Zacny, Patrick T. Taylor, Improved data reduction algorithm for the needle probe method applied to in-situ thermal conductivity measurements of lunar and planetary regolith, Planetary and Space Science 92 (2014) 49-56

23. Lam Dang, Wey H. Leong, Thermal conductivity probe - Part II - An experimental analysis, International Journal of Heat and Mass Transfer 86 (2015) 1004-1014.

24. V.R. Tarnawski, T. Momose, W.H. Leong, G. Bovesecchi, P. Coppa, Thermal conductivity of standard sands - Part 1 - dry state conditions, Int. J. Thermophys. 30 (2009) 949-968.

25. V.R. Tarnawski, T. Momose, W.H. Leong, Thermal conductivity of standard sands II. Saturated conditions, Int. J. Thermophys. 32 (2011) 984-1005. 